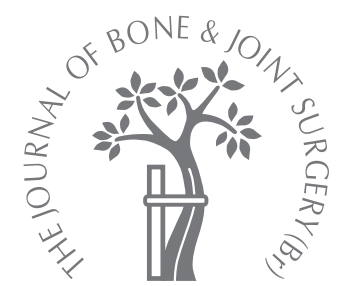

- CASE REPORT

\title{
Tibiofibular synostosis in a professional football player
}

\section{S. H. James, \\ E. C. Carpenter, J. A. Fairclough \\ From the University Hospital of Wales, Cardiff, Wales}

\author{
We present an unusual cause of anterior tibial pain in a 24-year-old professional \\ international football player who was found to have a synostosis of the middle-third of the \\ diaphysis of the tibia and fibula. \\ This is a rarely described phenomenon. Conservative treatment is the recommended \\ treatment of choice, but this failed in our patient. Resection produced resolution of \\ symptoms; he remained symptom-free three years later.
}

Pain in the anterior compartment of the lower leg is common in relation to athletic activities, and may be due to compartment syndrome, stress fractures and tibial stress syndrome. ${ }^{1}$

\section{Case report}

A 24-year-old professional football player of Afro-Caribbean origin presented with a history of left anterior shin pain for 18 months. The pain increased with exercise and was most troublesome after prolonged running in training. There were no paraesthesiae in the calf or foot. He was, however, able to cycle and to exercise with weights in the gymnasium without symptoms. There was no history of trauma.

Initially, the pain had presented after preseason training as an aching sensation in the calf, which had lasted for less than an hour after exercise. He was still able to play football but could not participate in full training. Following gait analysis he was provided with insoles on the assumption that his flexible pes planovalgus was the cause of the pain. However, the symptoms became more severe. He was treated by the club physiotherapist with ice applications after training and was instructed to perform a monitored coolingdown programme of exercise.

The symptoms worsened over a period of three months with the anterior calf pain occurring after only short periods of running and persisting for several hours after the cessation of activity. He was no longer able to complete training, or match sessions.

Clinical examination revealed a muscular athlete with no obvious wasting. His gait was normal. Alignment of the lower limbs was nor-

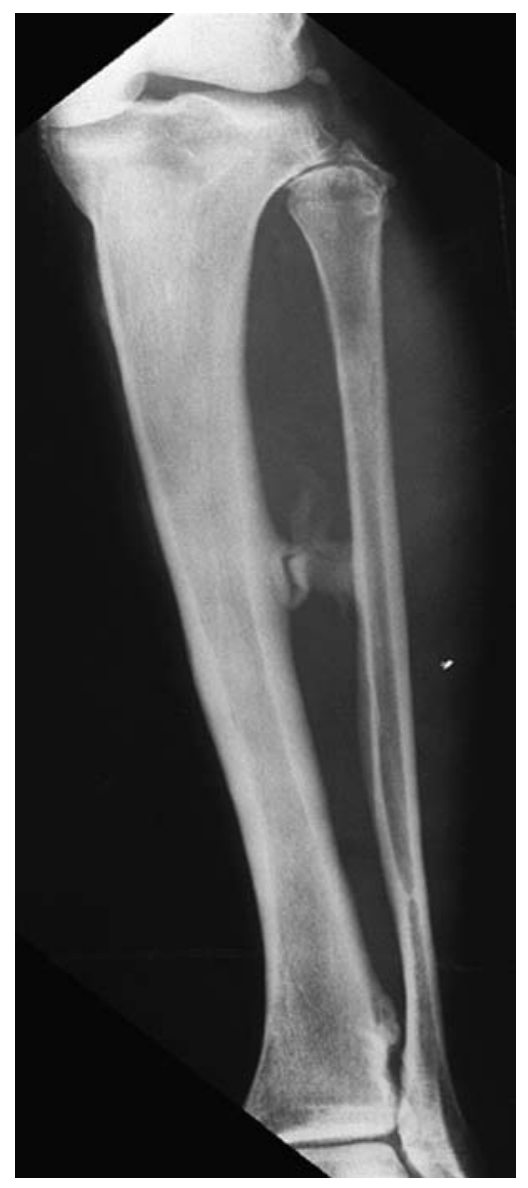

Fig. 1

Plain anteroposterior radiograph of the lower limb showing the bony synostosis.

mal apart from mild bilateral flexible planovalgus feet. There was no tenderness in the compartments of the lower leg or at the sites of origin and insertion of the muscles. The muscle 


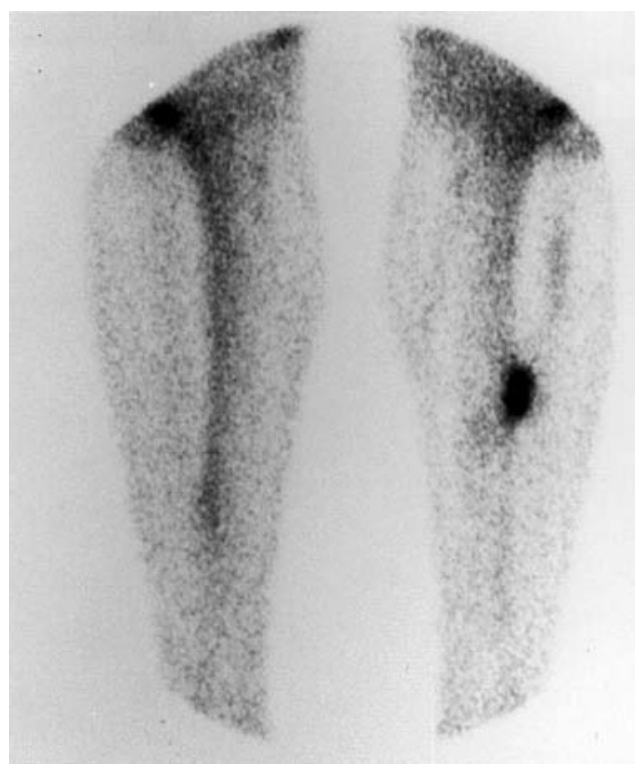

Fig. 2

Isotope bone scan showing increased activity at the level of the synostosis.

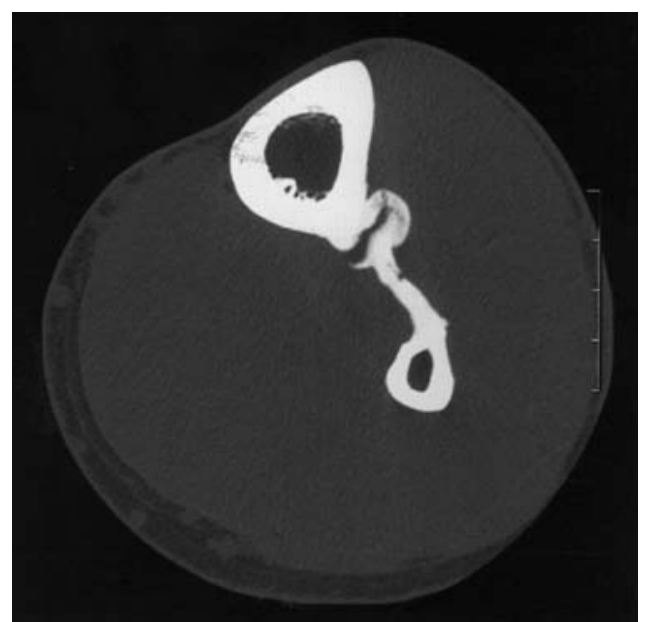

Fig. 3a

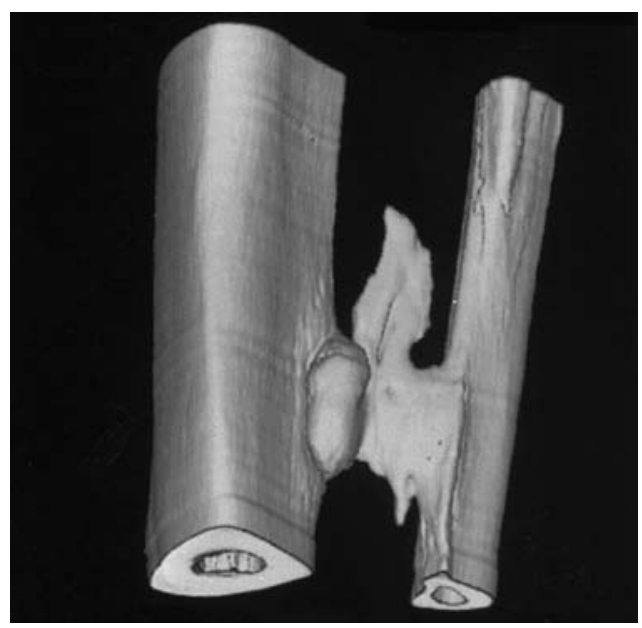

Fig. 3b

The bony bridge shown by a) axial CT and b) $3 \mathrm{D}$ CT reconstruction.
An isotope bone scan showed increased activity in the region of this synostosis (Fig. 2). Three-dimensional (3D) CT showed that the lesion extended along the interosseous membrane (Fig. 3).

Initial treatment consisted of reduced training and match play until the end of the season and then a gradual return to running and pre-season training. This failed to relieve the symptoms and resection of the bony bridge was undertaken through an anterolateral incision without a tourniquet. The findings were as seen on the 3D CT scan. The anterior muscles were reflected laterally to expose the intra-osseous region. The normal fibrous interosseous membrane had ossified into an irregular bony bridge between the posterior aspect of the fibula and the posteromedial surface of the tibia. The bony area was approximately $2 \mathrm{~mm}$ deep and $3 \mathrm{~cm}$ long. The distal dge of the ossified area was dissected from the fibrous interosseous tissue and carefully excised, avoiding the nearby neurovascular bundle, as far as the margins of the tibia and posterior fibula. Although the removal of the bone left a defect in the interosseous membrane of approximately $2.5 \mathrm{~cm}$, no herniation of adjacent muscle was seen at the completion of the dissection.
After operation, careful monitoring was undertaken to assess any increase in compartment pressures. After 24 hours he was discharged non-weight-bearing in a belowknee cast. After two weeks the cast was removed and progressive weight-bearing undertaken, with return to light training after six weeks. He was able to return to competitive football after three months.

Three years later there has been no recurrence of symptoms or radiological evidence of a return of the synostosis. $\mathrm{He}$ is still playing professional football at international level.

\section{Discussion}

Synostosis of the middle-third of the diaphysis of the tibia and fibula is rare and has not been described previously in football players. Similar conditions have been noted in runners and basketball players, all of whom presented with 
anterior shin pain for one or two years., ${ }^{2,3}$ Due to the chronicity of the symptoms in these cases the synostosis was attributed to an occult stress fracture or severe ligament sprain. In our patient there was no history of trauma and the short duration of the symptoms indicated that a stress fracture of the fibula was unlikely. The location of the synostosis suggested that the aetiology was that of a stress phenomenon to the interosseous membrane.

Depending on the location, synostoses may be asymptomatic, resulting from an accompanying deformity of the limb segment. ${ }^{4}$ Symptomatic synostoses of the lower limbs are a cause of pain and have been thought to be associated with stress fractures of the fibula. ${ }^{5}$ Several causes of synostosis have been described. Congenital types ${ }^{1}$ may be associated with multiple hereditary exostosis and typically present at an earlier age. Other causes include trauma, predominantly to the distal tibia and fibula ${ }^{6}$ and ankle. ${ }^{7-9}$ In these cases the synostosis is believed to result from a haematoma tracking between the two bones, and undergoing calcification on the interosseous membrane. Iatrogenic causes have also been described after tibial bone grafting. ${ }^{10}$

Resection of the synostosis provided a good result in our patient with resolution of the symptoms and a return to full sporting activity. A high risk of recurrence has been noted, particularly in cases of congenital or post-traumatic synostosis, specifically in the region of the distal tibiofibular joint. No particular surgical technique has been described which lessens the risk of recurrence, but it would appear to be logical to remove all the pathological bone which has replaced the normal fibrous tissue, thereby prohibiting movement between the tibia and fibula. Henry et al ${ }^{3}$ suggested that these lesions may be treated successfully by conservative measures. Our findings support those of previous reports ${ }^{1,2}$ which indicate that idiopathic mid-diaphyseal synostosis does not respond to conservative management and is best treated by resection.

No benefits in any form have been received or will be received from a commercial party related directly or indirectly to the subject of this article.

\section{References}

1. Håkan I, Magnusson HI, Westlin NE, et al. Abnormally decreased regional bone density in athletes with medial tibial stress syndrone. Am J Sports Med 2001;29:712-15.

2. Flandry F, Saunders RA. Tibiofibular synostosis: an unusual cause of shin splint-like pain. Am J Sports Med 1987;15:280-4

3. Henry JH, Anderson JA, Cothren CC. Tibiofibular synostosis in professional basketball players. Am J Sports Med 1993;21:519-622.

4. Wong K, Weiner DS. Proximal tibiofibular synostosis. Clin Orthop 1978;135:457.

5. Kottmeier SA, Hanks GA, Kalenak A. Fibular stress fracture associated with distal tibiofibular synostosis in an athlete: a case report and literature review. Clin Orthop 1992:281:195-8.

6. Munjal K, Kishan S, Sabharwal S. Posttraumatic pediatric distal tibiofibular synostosis: a case report. Foot Ankle Int 2004;25:429-33.

7. Albers GH, De Kort AF, Middendorf PR, van Dijk CN. Distal tibiofibular synostosis after ankle fracture: a 14-year follow-up study. J Bone Joint Surg [Br] 1996;78-B:250-2

8. McMaster JH, Scranton PE Jr. Tibiofibular synostosis: a cause of ankle disability. Clin Orthop 1975;111:172-4

9. Whiteside LA, Reynolds FC, Ellsasser JC. Tibiofibular synostosis in recurrent ankle strains in high performance athletes. Am J Sports Med 1978;6:204-8.

10. Skelley JW, Hardy AE. Results of bone grafts in the treatment of tibial fractures. Clin Orthop 1981:158:108-10. 